\title{
Management of Sucking Pest, Jassid (Amrasca devastans) and Thrips (Thrips palmi) on Lady's Finger (Abelmoschus esculentus L.) by using Safe Insecticides
}

\author{
Sunil Kumar Ghosh*
}

Department of Agricultural Entomology, B.C.K.V-Agricultural University, Kalyani, Nadia, West Bengal, 741235, India

*Corresponding author

\section{A B S T R A C T}

\begin{tabular}{|l|}
\hline K e y w o r d s \\
Dinotefuran, \\
Fipronil, \\
Imidacloprid, \\
Persistency, \\
Coccinellid
\end{tabular}

Lady's finger Abelmoschus esculentus (L.) Moench is an annual kharif vegetable crop belongs to the family Malvaceae. This crop is cultivated in various parts of tropical and sub-tropical areas of the world. In West Bengal, India it is cultivated in a commercial scale but its pest complex is very high which limit its production. Contribution jassid (Amrasca devastans) and thrips (Thrips palmi) as sucking pest in this case is of great importance. Three doses of dinotefuron 20 SG (@ 20,30 and 40 a.i. g /ha) two doses of imidacloprid 70 WG(@21 and 24.5 a.i. g /ha) and two doses of fipronil 5\% SC ( @ 25 and 37.5 a.i.g /ha) were applied to control jassid and thrips. From overall observation it was revealed that Dinotefuron 20 SG @ $40 \mathrm{~g}$ a.i./ha, Fipronil 5\% SC @ $37.5 \mathrm{~g}$ a.i./ha and imidacloprod 70 WG@24.5 g a.i/ha provided best suppression of jassid $(90.29 \%, 89.34 \%$ and $78.42 \%$ jassid population suppression respectively) and thrips (96.55\%, $96.01 \%$ and $85.37 \%$ thrips population suppression respectively). They are also safe insecticides to cocconellid predators. These insecticides may be recommended for farmers use to control sucking pest.

\section{Introduction}

Lady's finger Abelmoschus esculentus (L.) Moench is an annual kharif vegetable crop belongs to the family Malvaceae and grown in various parts of tropical and sub-tropical areas of the world. In the sub-Himalayan region of north east India lady'sfingers is cultivated at a commercial scale but insect pest damage constitutes a limiting factor in successful production (Ghosh, 2013; Ghosh, et al., 2013). Lady's finger is infested by a large number of sucking pest viz. jassid, thrips, mites, whitefly aphid etc. Contribution of jassid and thrips in this case is of great importance. Both nymphs and adults suck the sap from tender crop canopy, resulting in shriveling of leaves, retarded shoot development and finally the leaves fall-off, yield reduced significantly.

Ghosh and senapati (2003) reported that hopper/jassid population (4.63/leaf) was very high during April-May and positively correlated with temperature gradient, relative humidity and rainfall. Thrips population 
touched the peak during the third week of September (10.2/ 3 leaves) (Saini et al., 2017). Peak population of thrips (12.58 thrips/three leaves) was recorded in $18^{\text {th }}$ standard week (Priyadarshini et al., 2017). Subba and Ghosh (2016) reported that maximum level of thrips population on tomato was observed during $6^{\text {th }}$ to $20^{\text {th }}$ standard week.

Ghosh et al., (2005) reported that thrips population showed significant positive correlation $\quad(p=0.05)$ with temperature, relative humidity, and rainfall on eggplant. Laskar and Ghosh (2005) reported that thrips population ranged from 6.9-16.17 per leaf in untreated chilli field. Ghosh et al., (2007) reported that lady bird beetle / coccinellid beetle Menochilus sexmaculatus was an important predator of aphid and jassid and its feeding activity was found throughout the year. Coccinellid beetle Coccinella septempunctata as the generalized predatory agent has gained great scientific interest for biological control in West Bengal, India (Ghosh, 1999; Chakraborty and Ghosh, 2010).

For the effective control of jassid, thrips in vegetable field farmers usually use a lot of pesticides chemicals indiscriminately and frequently (Ghosh, 1999). Ghosh et al., (2009) reported that imidacloprid was found most effective (91.15\% control) against aphid three days after treatment. Mandal et al., (2016) reported that imidaclorprid was found most effective against thrips on som plant providing $75.18 \%$ suppression.

Most of the conventional chemicals are broad spectrum, persistent in nature and having long residual action. Ghosh and Chakraborty (2012) reported that pest control by using biocontrol agent is an important component of Integrated Pest Management and organic farming. So, there is need of search of bio- control agent in specific time that can break the resistance and become eco-friendly. Das et al., (2010) and Ghosh et at. (2012) reported that a rapid degradation of persistency was observed in Imidacloprid which had a greater importance as fruits and vegetables are consumed after little cooking. Subba et al., (2015) reported that acetamiprid was very effective against jassid recording more than $80 \%$ control. Imidacloprid was the most effective in providing more than $80 \%$ aphid suppression followed by azadirachtin (Ghosh et al., 2016). Most of the Conventional chemicals are broad spectrum, persistent in nature and having long residual action (Subba et al, 2017; Nayar, et.al., 1992). The objective of the study was to formulate suitable management of sucking pests of lady's finger with the use of some new safe molecules and less harmful to beneficial insects and environment.

\section{Materials and Methods}

\section{Period and location of the study}

The studies were done at A-B Block Farm of Bidhan Chandra Krishi Viswavidyalaya located at Kalyani, West Bengal, India during the year 2018-2019. The geographical position of the areas are $23^{\circ} \mathrm{N}$ latitude, $89^{\circ} \mathrm{E}$ longitude and 9.75 meter above mean sea level (Thakoor et al., 2020). The soil was gangetic alluvial soil (Entisol) with sandy clay loam texture, neutral in reaction with moderate in fertility (Priyadarsini et al., 2019). The soil type of the experimental field was sandy loam with $\mathrm{PH}$ range 5.75 to 6.5 and climate of this zone is subtropical humid having short winter spell during December January (Bala et al., 2015; Karmakar et al., 2017). The experimental plot was situated on upland with good irrigation and drainage facility. The soil has good water holding capacity. 


\section{Treatment details}

\begin{tabular}{|c|c|c|c|}
\hline \multicolumn{4}{|c|}{ Treatment details are as follows: } \\
\hline Treatment & $\begin{array}{c}\text { Dose } \\
\text { g or ml. a.i. / ha }\end{array}$ & $\begin{array}{c}\text { Dose } \\
\text { (g or ml/ha) }\end{array}$ & $\begin{array}{c}\text { Spray fluid } \\
\text { used }\end{array}$ \\
\hline$T_{1}$ : UTC & -- & -- & $\mathbf{5 0 0}$ \\
\hline$T_{2}$ : Dinotefuron 20 SG & 20 & 100 & $\mathbf{5 0 0}$ \\
\hline $\mathbf{T}_{\mathbf{3}}$ : Dinotefuron 20 SG & 30 & 150 & $\mathbf{5 0 0}$ \\
\hline $\mathbf{T}_{\mathbf{4}}$ : Dinotefuron 20 SG & 40 & 200 & $\mathbf{5 0 0}$ \\
\hline $\mathbf{T}_{\mathbf{5}}$ : Imidacloprid 70WG & 21 & 30 & $\mathbf{5 0 0}$ \\
\hline $\mathbf{T}_{\mathbf{6}}$ : Imidacloprod 70 WG & 24.5 & 35 & $\mathbf{5 0 0}$ \\
\hline $\mathbf{T}_{\mathbf{7}}$ : Fipronil 5\% SC & 25 & 500.0 & $\mathbf{5 0 0}$ \\
\hline T8: Fipronil 5\% SC & 37.5 & 750.0 & $\mathbf{5 0 0}$ \\
\hline
\end{tabular}

\section{Lay out of the experiment}

Season of experiment : Kharif-2018

Variety of lady's finger: Samrat

$\begin{array}{ll}\text { Date of sowing } & : 12.07 .2018 \\ \text { Plot size } & : 5 \mathrm{~m} \mathrm{X} \mathrm{5m} \\ \text { Spacing } & : \text { P-P X R-R = 5 cm X } \\ & 20 \mathrm{~cm}\end{array}$

Fertilizers $\quad$ : N: P: K @ 100:60:60

Design of experiment : Randomized Block

Design (RBD)

Number of replications : Three

Number of spraying : Two

Application date : First spray:

10.08.2018 and

second spray:

25.08.2018

Application method : ASPEE Knapsack

Sprayer with hollow cone nozzle

Harvesting : Multiple picking

Methodology for bio-efficacy data recording against pests

The data of target pests were recorded from randomly selected five plants in each plot. Observations of total number of jassid and thrips on lady's finger were recorded from five top young leaves of each plant per plot and converted to number of insect pest/leaf. First count was taken one day before first spray and post treatment counts were recorded on 3, 7, 10 and 14 days after each spray. All the observations were recorded with the help of a hand lens (10X).

The population of natural enemies (coccinellid) was also recorded from 5 randomly selected plants on 10 days after each spray. Reduction of insect population in different treatments over control was used as an indicator of insecticidal efficacy which was calculated from the following formula (Abbott, 1925):

$$
\begin{aligned}
& P o-P c \\
& \mathrm{Pt}=\mathrm{-PC}
\end{aligned}
$$

Where, $\mathrm{Pt}=$ Corrected mortality, $\mathrm{Po}=$ Observed mortality and $\mathrm{Pc}=$ Control mortality.

Data were analyzed by using INDO-STATsoftware for analysis of variance following randomized block design (RBD) treatment means were separated by applying CD Test (critical difference) at $5 \%$ level of significance.

\section{Results and Discussion}

Three doses of dinotefuron 20 SG (@ 20,30 and 40 a.i. g /ha) two doses of imidacloprid 
70 WG(@ 21 and 24.5 a.i. g /ha) and two doses of fipronil 5\% SC (@ 25 and 37.5 a.i.g /ha) were sprayed to work out their efficacy against jassid and thrips.

One treatment of untreated control was taken for observing natural infestation of target pests. Two round spraying has been done where first round was initiated during second week of July and subsequent spraying has been done at 15 days interval. The data on the result has been presented in table 1-4 and the natural enemy population has been presented in table-5.

\section{Efficacy evaluation of insecticides against jassid (A. devastans)}

Data presented in Table 1 and 2 revealed that, all the insecticide treatments significantly reduced the infestation of jassid as compared to untreated control (5.06-7.62 jassid/leaf).

From the two round spray it was revealed that dinotefuron 20 SG @ $40 \mathrm{~g}$ a.i./ha provided best suppression of jassid population (first spray $92.59 \%$ and second spray $87.99 \%$ with a mean of $90.29 \%$ suppression) closely followed by fipronil 5\% SC @ $37.5 \mathrm{~g}$ a.i./ha (first spray $92.65 \%$ and second spray 86.03 $\%$ with a mean of $89.34 \%$ suppression) and imidacloprod $70 \mathrm{WG} @ 24.5 \mathrm{~g}$ a.i/ha (first spray $84.29 \%$ and second spray $72.55 \%$ with a mean of $78.42 \%$ suppression).

Three days after first spraying lowest population was recorded in Dinotefuron 20 SG @ $40 \mathrm{~g}$ a.i./ha treated plot $(0.34 \mathrm{jassid} / \mathrm{L})$ closely followed by fipronil 5\% SC @ $37.5 \mathrm{~g}$ a.i./ha (0.36 jassid/L) and imidacloprod 70 WG @ $24.5 \mathrm{~g}$ a.i/ha (0.78 jassid/L).

There were no significant differences among these three treatments. Similar trend was followed 7 days, 10 days and 14 days after first spraying. Three days after second spraying lowest population was recorded in dinotefuron 20 SG @ $40 \mathrm{~g}$ a.i./ha treated plot $(0.12 \mathrm{jassid} / \mathrm{L})$ closely followed by Fipronil 5\% SC @ $37.5 \mathrm{~g}$ a.i./ha (0.13 jassid/L) and imidacloprod 70 WG @ 24.5 g a.i/ha $(0.45$ jassid/L).

There were no significant differences among these three treatments. Similar trend was followed 7 days after second spraying. Ten days after second spraying lowest population was recorded in dinotefuron 20 SG @ $40 \mathrm{~g}$ a.i./ha treated plot $(0.78 \mathrm{jassid} / \mathrm{L})$ closely followed by fipronil 5\% SC @ $37.5 \mathrm{~g}$ a.i./ha (0.79 jassid/L).

There were no significant differences between these two treatments. Similar trend was followed 14 days after second spraying.

\section{Efficacy evaluation of insecticides against thrips (Thrips palmi)}

Data presented in Table 3 and 4 revealed that, all the insecticide treatments significantly reduced the infestation of thrips as compared to untreated control (5.46-8.33 thrips/leaf).

From the two round spray it is revealed that dinotefuron $20 \mathrm{SG} @ 40 \mathrm{~g}$ a.i./ha provided best suppression of thrips population (first spray $96.32 \%$ and second spray $96.78 \%$ with a mean of $96.55 \%$ suppression) closely followed by fipronil 5\% SC @ $37.5 \mathrm{~g}$ a.i./ha (first spray $96.17 \%$ and second spray 95.86 $\%$ with a mean of $96.01 \%$ suppression) and imidacloprod 70 WG @ $24.5 \mathrm{~g}$ a.i/ha (first spray $88.96 \%$ and second spray $81.78 \%$ with a mean of $85.37 \%$ suppression).

Three days after first spraying lowest population was recorded in Dinotefuron 20 SG @ $40 \mathrm{~g}$ a.i./ha treated plot (0.04 thrips/L) closely followed by fipronil 5\% SC @ $37.5 \mathrm{~g}$ a.i./ha (0.06 thrips/L) and imidacloprod 70 WG @ $24.5 \mathrm{~g}$ a.i/ha (0.57 thrips/L). There were no significant differences among these three treatments. 
Table.1 Effect of insecticides on the population abundance of jassid in lady's finger, Kharif-2018 ( $1^{\text {st }}$ spray)

\begin{tabular}{|c|c|c|c|c|c|c|c|c|c|}
\hline \multirow[t]{3}{*}{ Treatments } & \multirow{2}{*}{\multicolumn{2}{|c|}{ Dose/ha }} & \multirow{3}{*}{$\begin{array}{c}\text { Pre- } \\
\text { treatment } \\
\text { Count } \\
(\text { jassid/L) }\end{array}$} & \multirow{2}{*}{\multicolumn{4}{|c|}{$\begin{array}{c}\text { Mean no. of jassid/leaf } \\
1^{\text {st }} \text { Spray }\end{array}$}} & \multirow[t]{3}{*}{ Post mean } & \multirow{3}{*}{$\begin{array}{c}\% \\
\text { reduction } \\
\text { over control }\end{array}$} \\
\hline & & & & & & & & & \\
\hline & $\begin{array}{l}\text { (g.or ml } \\
\text { a.i.) }\end{array}$ & $\begin{array}{l}\text { Formulatio } \\
\mathrm{n}(\mathrm{g} \text { or } \mathrm{ml})\end{array}$ & & 3 DAS & 7DAS & 10 DAS & 14 DAS & & \\
\hline \multirow[t]{2}{*}{$\mathrm{T}_{1}: \mathrm{UTC}$} & \multirow[t]{2}{*}{-- } & \multirow{2}{*}{--} & 4.12 & 4.78 & 5.34 & 6.54 & 6.98 & \multirow[t]{2}{*}{5.06} & \multirow[t]{2}{*}{0.00} \\
\hline & & & $(2.54)$ & $(2.70)$ & $(2.82)$ & $(3.07)$ & $(3.15)$ & & \\
\hline \multirow[t]{2}{*}{$\mathrm{T}_{2}:$ Dinotefuron $20 \mathrm{SG}$} & \multirow[t]{2}{*}{20} & \multirow[t]{2}{*}{100} & 4.23 & 2.67 & 2.98 & 3.23 & 3.67 & \multirow[t]{2}{*}{2.83} & \multirow[t]{2}{*}{45.62} \\
\hline & & & $(2.57)$ & $(2.14)$ & $(2.24)$ & $(2.31)$ & $(2.43)$ & & \\
\hline \multirow[t]{2}{*}{$\mathrm{T}_{3}$ : Dinotefuron $20 \mathrm{SG}$} & \multirow[t]{2}{*}{30} & \multirow[t]{2}{*}{150} & 4.33 & 1.01 & 1.23 & 1.76 & 2.02 & \multirow[t]{2}{*}{1.12} & \multirow[t]{2}{*}{78.96} \\
\hline & & & $(2.59)$ & $(1.51)$ & (1.62) & $(1.84)$ & (1.93) & & \\
\hline \multirow{2}{*}{$\mathrm{T}_{4}:$ Dinotefuron $20 \mathrm{SG}$} & \multirow[t]{2}{*}{40} & \multirow[t]{2}{*}{200} & 4.34 & 0.34 & 0.45 & 0.78 & 1.03 & \multirow[t]{2}{*}{0.40} & \multirow[t]{2}{*}{92.59} \\
\hline & & & $(2.59)$ & $(1.09)$ & $(1.18)$ & $(1.39)$ & $(1.52)$ & & \\
\hline \multirow[t]{2}{*}{$\mathrm{T}_{5}:$ Imidacloprid 70WG } & \multirow[t]{2}{*}{21} & \multirow[t]{2}{*}{30} & 4.65 & 1.25 & 1.45 & 1.87 & 2.01 & \multirow[t]{2}{*}{1.35} & \multirow[t]{2}{*}{76.38} \\
\hline & & & $(2.67)$ & (1.63) & $(1.71)$ & (1.88) & (1.93) & & \\
\hline \multirow[t]{2}{*}{$\mathrm{T}_{6}:$ Imidacloprod $70 \mathrm{WG}$} & \multirow[t]{2}{*}{24.5} & \multirow[t]{2}{*}{35} & 4.56 & 0.78 & 0.98 & 1.45 & 1.68 & 0.88 & 84.29 \\
\hline & & & $(2.65)$ & (1.39) & $(1.50)$ & $(1.71)$ & $(1.81)$ & & \\
\hline $\mathrm{T}_{7}:$ Fipronil 5\% SC & 25 & 500 & 4.54 & 1.04 & 1.22 & 1.78 & 2.09 & 1.13 & 79.73 \\
\hline & & & $(2.64)$ & $(1.53)$ & $(1.61)$ & $(1.84)$ & $(1.96)$ & & \\
\hline $\mathrm{T}_{8}:$ Fipronil $5 \% \mathrm{SC}$ & 37.5 & 750 & 4.65 & 0.36 & 0.48 & 0.79 & 1.05 & 0.42 & 92.65 \\
\hline & & & $(2.66)$ & $(1.10)$ & $(1.19)$ & $(1.39)$ & $(1.52)$ & & \\
\hline & & & -- & 0.16 & 0.17 & 0.22 & 0.21 & & - \\
\hline C.D & & & NS & 0.51 & 0.54 & 0.68 & 0.66 & & - \\
\hline
\end{tabular}

Figures in parentheses indicate $\sqrt{X}+0.5$ transformed value, L=leaf, DAS: Days after spraying 
Table.2 Effect of insecticides on the population abundance of jassid in lady's finger, Kharif-2018 ( $2^{\text {nd }}$ spray)

\begin{tabular}{|c|c|c|c|c|c|c|c|c|c|}
\hline \multirow[t]{3}{*}{ Treatments } & \multirow{2}{*}{\multicolumn{2}{|c|}{ Dose/ha }} & \multirow{3}{*}{$\begin{array}{c}\text { Pre- } \\
\text { treatment } \\
\text { Count } \\
\text { (jassid/L }\end{array}$} & \multicolumn{4}{|c|}{ Mean no. of jassid/leaf } & \multirow[t]{3}{*}{ Post mean } & \multirow{3}{*}{$\begin{array}{c}\% \\
\text { reduction } \\
\text { over contro }\end{array}$} \\
\hline & & & & \multicolumn{4}{|c|}{$2^{\text {nd }}$ Spray } & & \\
\hline & $\begin{array}{l}\text { (g.or ml } \\
\text { a.i.) }\end{array}$ & $\begin{array}{c}\text { Formulat } \\
\text { ion }(\mathrm{g} \text { or } \\
\mathrm{ml})\end{array}$ & & 3 DAS & 7 DAS & 10 DAS & 14 DAS & & \\
\hline \multirow[t]{2}{*}{$\mathrm{T}_{1}: \mathbf{U T C}$} & \multirow[t]{2}{*}{--} & \multirow[t]{2}{*}{--} & 4.12 & 7.34 & 7.89 & 8.34 & 8.79 & \multirow[t]{2}{*}{7.62} & \multirow[t]{2}{*}{0.00} \\
\hline & & & $(2.54)$ & $(3.22)$ & $(3.32)$ & $(3.40)$ & $(3.47)$ & & \\
\hline \multirow[t]{2}{*}{$\mathrm{T}_{2}$ : Dinotefuron $20 \mathrm{SG}$} & \multirow[t]{2}{*}{20} & \multirow[t]{2}{*}{100} & 4.23 & 1.12 & 1.45 & 1.87 & 2.03 & \multirow[t]{2}{*}{1.29} & \multirow[t]{2}{*}{67.91} \\
\hline & & & $(2.57)$ & $(1.57)$ & $(1.71)$ & $(1.88)$ & (1.93) & & \\
\hline \multirow{2}{*}{$\mathrm{T}_{3}$ : Dinotefuron $20 \mathrm{SG}$} & \multirow[t]{2}{*}{30} & \multirow[t]{2}{*}{150} & 4.33 & 0.78 & 1.11 & 1.32 & 1.97 & \multirow[t]{2}{*}{0.95} & \multirow[t]{2}{*}{57.12} \\
\hline & & & $(2.59)$ & $(1.39)$ & $(1.56)$ & $(1.66)$ & $(1.91)$ & & \\
\hline \multirow[t]{2}{*}{$\mathrm{T}_{4}:$ Dinotefuron $20 \mathrm{SG}$} & \multirow[t]{2}{*}{40} & \multirow[t]{2}{*}{200} & 4.34 & 0.12 & 0.15 & 0.28 & 0.43 & \multirow[t]{2}{*}{0.14} & \multirow[t]{2}{*}{87.99} \\
\hline & & & $(2.59)$ & $(0.86)$ & $(0.90)$ & $(1.04)$ & $(1.17)$ & & \\
\hline \multirow[t]{2}{*}{$\mathrm{T}_{5}:$ Imidacloprid 70WG } & \multirow[t]{2}{*}{21} & \multirow[t]{2}{*}{30} & 4.65 & 1.03 & 1.13 & 1.78 & 2.01 & \multirow[t]{2}{*}{1.08} & \multirow[t]{2}{*}{50.75} \\
\hline & & & $(2.67)$ & $(1.52)$ & $(1.57)$ & $(1.84)$ & $(1.93)$ & & \\
\hline \multirow{2}{*}{$\mathrm{T}_{6}$ : Imidacloprod $70 \mathrm{WG}$} & \multirow[t]{2}{*}{24.5} & 35 & 4.56 & 0.45 & 0.67 & 0.98 & 1.32 & 0.56 & 72.55 \\
\hline & & & $(2.65)$ & $(1.18)$ & $(1.33)$ & $(1.50)$ & $(1.66)$ & & \\
\hline $\mathrm{T}_{7}:$ Fipronil $5 \% \mathrm{SC}$ & 25 & 500 & 4.54 & 0.83 & 1.15 & 1.43 & 1.98 & 0.99 & 56.58 \\
\hline & & & $(2.64)$ & $(1.42)$ & $(1.58)$ & $(1.71)$ & $(1.92)$ & & \\
\hline $\mathrm{T}_{8}:$ Fipronil $5 \% \mathrm{SC}$ & 37.5 & 750 & 4.65 & 0.13 & 0.19 & 0.30 & 0.45 & 0.16 & 86.03 \\
\hline & & & $(2.66)$ & $(0.86)$ & $(0.94)$ & $(1.05)$ & $(1.17)$ & & \\
\hline $\mathbf{S}$ & & & -- & 0.15 & 0.17 & 0.16 & 0.18 & & - \\
\hline C.D & & & NS & 0.46 & 0.53 & 0.51 & 0.56 & & - \\
\hline
\end{tabular}

Figures in parentheses indicate $\sqrt{X}+0.5$ transformed value, L=leaf, DAS: Days after spraying 
Table.3 Effect of insecticides on the population abundance of thrips in lady's finger, Kharif-2018 ( $1^{\text {st }}$ spray)

\begin{tabular}{|c|c|c|c|c|c|c|c|c|c|}
\hline \multirow[t]{3}{*}{ Treatments } & \multirow{2}{*}{\multicolumn{2}{|c|}{ Dose/ha }} & \multirow{3}{*}{$\begin{array}{c}\text { Pre- } \\
\text { treatment } \\
\text { Count } \\
\text { (thrips/L) }\end{array}$} & \multicolumn{4}{|c|}{ Mean no. of thrips/leaf } & \multirow[t]{3}{*}{ Post mean } & \multirow{3}{*}{$\begin{array}{c}\% \\
\text { reduction } \\
\text { over control }\end{array}$} \\
\hline & & & & \multicolumn{4}{|c|}{$1^{\text {st }}$ Spray } & & \\
\hline & $\begin{array}{l}\text { (g.or ml } \\
\text { a.i.) }\end{array}$ & $\begin{array}{l}\text { Formulati } \\
\text { on }(\mathrm{g} \text { or } \\
\mathrm{ml})\end{array}$ & & 3 DAS & 7 DAS & 10 DAS & 14 DAS & & \\
\hline \multirow[t]{2}{*}{$\mathrm{T}_{1}: \mathrm{UTC}$} & \multirow[t]{2}{*}{--} & \multirow[t]{2}{*}{--} & 3.79 & 5.03 & 5.88 & 7.25 & 7.67 & \multirow[t]{2}{*}{5.46} & \multirow[t]{2}{*}{0.00} \\
\hline & & & $(2.46)$ & $(2.75)$ & $(2.94)$ & $(3.20)$ & $(3.28)$ & & \\
\hline \multirow[t]{2}{*}{$\mathrm{T}_{2}$ : Dinotefuron $20 \mathrm{SG}$} & \multirow[t]{2}{*}{20} & \multirow[t]{2}{*}{100} & 3.91 & 2.66 & 3.23 & 3.53 & 3.95 & \multirow[t]{2}{*}{2.95} & \multirow[t]{2}{*}{47.74} \\
\hline & & & $(2.49)$ & $(2.14)$ & $(2.31)$ & $(2.39)$ & $(2.50)$ & & \\
\hline \multirow{2}{*}{$\mathrm{T}_{3}$ : Dinotefuron $20 \mathrm{SG}$} & \multirow[t]{2}{*}{30} & \multirow[t]{2}{*}{150} & 4.02 & 0.80 & 1.26 & 1.88 & 2.09 & \multirow[t]{2}{*}{1.03} & \multirow[t]{2}{*}{82.25} \\
\hline & & & $(2.52)$ & $(1.40)$ & $(1.63)$ & $(1.88)$ & $(1.96)$ & & \\
\hline \multirow[t]{2}{*}{$\mathrm{T}_{4}:$ Dinotefuron $20 \mathrm{SG}$} & \multirow[t]{2}{*}{40} & \multirow[t]{2}{*}{200} & 4.03 & 0.04 & 0.39 & 0.78 & 0.98 & \multirow[t]{2}{*}{0.21} & \multirow[t]{2}{*}{96.32} \\
\hline & & & $(2.52)$ & $(0.72)$ & $(1.13)$ & $(1.39)$ & $(1.50)$ & & \\
\hline \multirow[t]{2}{*}{$\mathrm{T}_{5}:$ Imidacloprid 70WG } & \multirow[t]{2}{*}{21} & \multirow[t]{2}{*}{30} & 4.38 & 1.07 & 1.51 & 2.00 & 2.08 & \multirow[t]{2}{*}{1.29} & \multirow[t]{2}{*}{79.62} \\
\hline & & & $(2.60)$ & $(1.54)$ & $(1.74)$ & $(1.93)$ & $(1.95)$ & & \\
\hline \multirow{2}{*}{$\mathrm{T}_{6}:$ Imidacloprod $70 \mathrm{WG}$} & \multirow[t]{2}{*}{24.5} & 35 & 4.28 & 0.57 & 0.79 & 1.23 & 1.92 & 0.68 & 88.96 \\
\hline & & & $(2.58)$ & $(1.26)$ & $(1.40)$ & $(1.62)$ & $(1.90)$ & & \\
\hline $\mathrm{T}_{7}:$ Fipronil $5 \% \mathrm{SC}$ & 25 & 500 & 4.26 & 0.83 & 1.25 & 1.90 & 2.17 & 1.04 & 83.05 \\
\hline & & & $(2.57)$ & $(1.42)$ & $(1.63)$ & $(1.89)$ & $(1.98)$ & & \\
\hline $\mathrm{T}_{8}:$ Fipronil $5 \% \mathrm{SC}$ & 37.5 & 750 & 4.38 & 0.06 & 0.42 & 0.79 & 1.00 & 0.24 & 96.17 \\
\hline & & & $(2.59)$ & $(0.75)$ & $(1.15)$ & $(1.39)$ & $(1.50)$ & & \\
\hline & & & -- & 0.19 & 0.15 & 0.17 & 0.22 & & - \\
\hline C.D & & & NS & 0.54 & 0.43 & 0.47 & 0.62 & & - \\
\hline
\end{tabular}

Figures in parentheses indicate $\sqrt{\mathrm{X}}+0.5$ transformed value, $\mathrm{L}=$ leaf, DAS: Days after spraying 
Table.4 Effect of insecticides on the population abundance of thrips in lady'sfinger, Kharif-2018 ( $2^{\text {nd }}$ spray)

\begin{tabular}{|c|c|c|c|c|c|c|c|c|c|}
\hline \multirow[t]{3}{*}{ Treatments } & \multirow{2}{*}{\multicolumn{2}{|c|}{ Dose/ha }} & \multirow{3}{*}{$\begin{array}{c}\text { Pre- } \\
\text { treatment } \\
\text { Count } \\
\text { (thrips/L) }\end{array}$} & \multicolumn{4}{|c|}{ Mean no. of thrips/leaf } & \multirow[t]{3}{*}{ Post mean } & \multirow{3}{*}{$\begin{array}{c}\% \\
\text { reduction } \\
\text { over contro }\end{array}$} \\
\hline & & & & \multicolumn{4}{|c|}{$2^{\text {nd }}$ Spray } & & \\
\hline & $\begin{array}{l}\text { (g.or ml } \\
\text { a.i.) }\end{array}$ & $\begin{array}{c}\text { Formulat } \\
\text { ion }(\mathrm{g} \text { or } \\
\mathrm{ml})\end{array}$ & & 3 DAS & 7 DAS & 10 DAS & 14 DAS & & \\
\hline \multirow[t]{2}{*}{$\mathrm{T}_{1}: \mathbf{U T C}$} & \multirow[t]{2}{*}{--} & \multirow[t]{2}{*}{--} & 3.79 & 7.91 & 8.75 & 9.28 & 9.70 & \multirow[t]{2}{*}{8.33} & \multirow[t]{2}{*}{0.00} \\
\hline & & & $(2.46)$ & $(3.32)$ & $(3.47)$ & $(3.56)$ & $(3.62)$ & & \\
\hline \multirow[t]{2}{*}{$\mathrm{T}_{2}$ : Dinotefuron $20 \mathrm{SG}$} & \multirow[t]{2}{*}{20} & \multirow[t]{2}{*}{100} & 3.91 & 1.56 & 1.87 & 2.00 & 2.45 & \multirow[t]{2}{*}{1.72} & \multirow[t]{2}{*}{60.03} \\
\hline & & & $(2.49)$ & $(1.76)$ & $(1.88)$ & $(1.93)$ & $(2.08)$ & & \\
\hline \multirow[t]{2}{*}{$\mathrm{T}_{3}$ : Dinotefuron $20 \mathrm{SG}$} & \multirow[t]{2}{*}{30} & \multirow[t]{2}{*}{150} & 4.02 & 0.54 & 0.89 & 1.12 & 1.54 & \multirow[t]{2}{*}{0.71} & \multirow[t]{2}{*}{68.57} \\
\hline & & & $(2.52)$ & $(1.24)$ & $(1.45)$ & $(1.57)$ & $(1.75)$ & & \\
\hline \multirow[t]{2}{*}{$\mathrm{T}_{4}:$ Dinotefuron $20 \mathrm{SG}$} & \multirow[t]{2}{*}{40} & \multirow[t]{2}{*}{200} & 4.03 & 0.02 & 0.05 & 0.21 & 0.30 & \multirow[t]{2}{*}{0.03} & \multirow[t]{2}{*}{96.78} \\
\hline & & & $(2.52)$ & $(0.65)$ & $(0.73)$ & $(0.97)$ & $(1.06)$ & & \\
\hline \multirow[t]{2}{*}{$\mathrm{T}_{5}:$ Imidacloprid 70WG } & \multirow[t]{2}{*}{21} & \multirow[t]{2}{*}{30} & 4.38 & 0.45 & 0.78 & 1.32 & 1.78 & \multirow[t]{2}{*}{0.62} & \multirow[t]{2}{*}{72.78} \\
\hline & & & $(2.60)$ & $(1.18)$ & $(1.39)$ & $(1.66)$ & $(1.84)$ & & \\
\hline \multirow{2}{*}{$\mathrm{T}_{6}$ : Imidacloprod $70 \mathrm{WG}$} & \multirow[t]{2}{*}{24.5} & 35 & 4.28 & 0.34 & 0.42 & 0.57 & 0.89 & 0.38 & 81.78 \\
\hline & & & $(2.58)$ & $(1.09)$ & $(1.16)$ & $(1.26)$ & $(1.45)$ & & \\
\hline $\mathrm{T}_{7}:$ Fipronil $5 \% \mathrm{SC}$ & 25 & 500 & 4.26 & 0.59 & 0.96 & 1.19 & 1.62 & 0.78 & 67.12 \\
\hline & & & $(2.57)$ & $(1.28)$ & $(1.49)$ & $(1.60)$ & $(1.78)$ & & \\
\hline $\mathrm{T}_{8}:$ Fipronil $5 \% \mathrm{SC}$ & 37.5 & 750 & 4.38 & 0.03 & 0.06 & 0.24 & 0.33 & 0.05 & 95.86 \\
\hline & & & $(2.59)$ & $(0.67)$ & $(0.74)$ & $(0.99)$ & $(1.07)$ & & \\
\hline $\mathbf{S}$ & & & -- & 0.15 & 0.20 & 0.16 & 0.18 & & - \\
\hline C.D & & & NS & 0.43 & 0.38 & 0.45 & 0.51 & & - \\
\hline
\end{tabular}

Figures in parentheses indicate $\sqrt{\mathrm{X}}+0.5$ transformed value, $\mathrm{L}=$ leaf, DAS: Days after spraying 
Table.5 Effect of insecticides on the population abundance of predator in lady's finger Kharif-2018

\begin{tabular}{|c|c|c|c|c|c|c|}
\hline \multirow[t]{2}{*}{ Treatments } & \multicolumn{2}{|c|}{ Dose/ha } & \multirow{2}{*}{$\begin{array}{c}\text { Pre-treatment } \\
\text { Count } \\
\text { Coccinellids } \\
\text { (Number/p) }\end{array}$} & \multicolumn{3}{|c|}{$\begin{array}{c}\text { Coccinellids } \\
\text { (Number/plant) }\end{array}$} \\
\hline & (g.or $\mathrm{ml}$ a.i.) & $\begin{array}{l}\text { Formulation (g } \\
\quad \text { or } \mathrm{ml})\end{array}$ & & $\begin{array}{c}\text { 10DAS } \\
\left(1^{\text {st }} \text { spray }\right)\end{array}$ & $\begin{array}{c}10 \text { DAS } \\
\left(2^{\text {nd }} \text { spray }\right)\end{array}$ & $\begin{array}{c}\text { Mean of } 1^{\text {st }} \text { and } \\
2^{\text {nd }} \text { spray }\end{array}$ \\
\hline \multirow[t]{2}{*}{$\mathrm{T}_{1}: \mathrm{UTC}$} & \multirow[t]{2}{*}{--} & \multirow[t]{2}{*}{--} & 4.53 & 4.76 & 4.98 & 4.87 \\
\hline & & & $(2.64)$ & $(2.69)$ & $(2.74)$ & \\
\hline \multirow[t]{2}{*}{$\mathrm{T}_{2}:$ Dinotefuron $20 \mathrm{SG}$} & \multirow[t]{2}{*}{20} & \multirow[t]{2}{*}{100} & 4.34 & 4.33 & 4.31 & 4.32 \\
\hline & & & $(2.59)$ & $(2.59)$ & $(2.59)$ & \\
\hline \multirow[t]{2}{*}{$\mathrm{T}_{3}$ : Dinotefuron $20 \mathrm{SG}$} & \multirow[t]{2}{*}{30} & \multirow[t]{2}{*}{150} & 4.34 & 4.32 & 4.12 & 4.22 \\
\hline & & & $(2.59)$ & $(2.59)$ & $(2.54)$ & \\
\hline \multirow[t]{2}{*}{$\mathrm{T}_{4}$ : Dinotefuron $20 \mathrm{SG}$} & \multirow[t]{2}{*}{40} & \multirow[t]{2}{*}{200} & 5.00 & 4.64 & 4.32 & 4.48 \\
\hline & & & $(2.75)$ & $(2.66)$ & $(2.59)$ & \\
\hline \multirow[t]{2}{*}{$\mathrm{T}_{5}:$ Imidacloprid 70WG } & \multirow[t]{2}{*}{21} & \multirow[t]{2}{*}{30} & 4.32 & 4.28 & 4.13 & 4.20 \\
\hline & & & $(2.59)$ & $(2.54)$ & $(2.54)$ & \\
\hline \multirow[t]{2}{*}{$\mathrm{T}_{6}$ : Imidacloprod $70 \mathrm{WG}$} & \multirow[t]{2}{*}{24.5} & \multirow[t]{2}{*}{35} & 4.23 & 4.22 & 4.18 & 4.20 \\
\hline & & & $(2.57)$ & $(2.57)$ & $(2.48)$ & \\
\hline \multirow[t]{2}{*}{$\mathrm{T}_{7}$ : Fipronil 5\% SC } & \multirow[t]{2}{*}{25} & \multirow[t]{2}{*}{500} & 4.65 & 4.12 & 4.34 & 4.23 \\
\hline & & & $(2.67)$ & $(2.54)$ & $(2.59)$ & \\
\hline \multirow[t]{2}{*}{$\mathrm{T}_{8}$ : Fipronil 5\% SC } & \multirow[t]{2}{*}{37.5} & \multirow[t]{2}{*}{750} & 4.21 & 4.15 & 4.15 & 4.15 \\
\hline & & & $(2.55)$ & $(2.53)$ & $(2.53)$ & \\
\hline \multicolumn{3}{|c|}{ C.D $(p<0.05)$} & NS & NS & NS & -- \\
\hline
\end{tabular}

Figures in parentheses indicate $\sqrt{X}+0.5$ transformed value, $P=$ plant, DAS: Days after spraying 
Similar trend was followed 7 days and 10 days after first spraying. Fourteen days after first spraying lowest population was recorded in dinotefuron $20 \mathrm{SG} @ 40 \mathrm{~g}$ a.i./ha treated plot (0.98 thrips/L) closely followed by fipronil 5\% $\quad$ SC @ 37.5 g a.i./ha (1.00 thrips/L). There were no significant differences between these two treatments. But these two treatment were significant different from imidacloprid treatment.

Three days after second spraying lowest population was recorded in dinotefuron $20 \mathrm{SG}$ (a) $40 \mathrm{~g}$ a.i./ha treated plot (0.02 thrip/L) closely followed by Fipronil 5\% SC @ $37.5 \mathrm{~g}$ a.i./ha (0.03 thrips/L) and imidacloprod 70 WG @ 24.5 g a.i/ha (0.34 thrips/L). There were no significant differences among these three treatments.

Similar trend was followed 7 days and 10 days after second spraying. Fourteen days after second spraying lowest population was recorded in dinotefuron $20 \mathrm{SG} @ 40 \mathrm{~g}$ a.i./ha treated plot $(0.30$ thrips/L) closely followed by fipronil 5\% SC @ $37.5 \mathrm{~g}$ a.i./ha (0.33thrips/L). There were no significant differences between these two treatments. But these two treatment were significant different from imidacloprid treatment.

\section{Effect of insecticides on natural enemies of lady's finger ecosystem}

During the period of study, natural enemy fauna were found included several species of coccinellid beetles and their grubs (Coccinella transversalis, $\quad$ C. septempunctata, Cheilomenes sexmaculata and Micraspis discolor), larvae of syrphid fly, chrysopids (Chrysoperla spp.) and spiders. Among these only coccinellids were observed frequently, whereas, the population of others were scanty. The data on coccinellid on the crop before and after treatment with different chemicals show that, none of the insecticidal treatments significantly reduced the population of coccinellid natural enemies than the untreated control (Table 5).

From overall observation it was found that Dinotefuron20 SG @40 g a.i./ha, Fipronil 5\% SC @ $37.5 \mathrm{~g}$ a.i./ha and imidacloprod 70 WG @ $24.5 \mathrm{~g}$ a.i/ha provided best suppression of jassid and thrips population. There were no significant differences among these treatments. These findings were supported by some research works. Thakoor Pavan et al., (2019) reported that maximum tomato sucking pest population reduction was found in the imidacloprid. Ghosh (2013) reported that Imidacloprid 17.8 SL resulted in the best suppression of sucking hopper population $(91.89 \%)$. Ghosh and Chakraborty (2015) reported that imidacloprid was found the most effective treatment for controlling lady'sfinger jassids, followed by the microbial insecticide spinosad. Ghosh (2020) reported that lower percent reduction of jassid was observed in the plots treated with fipronil 5\% SC than imidacloprid. Priyadarshini et al., (2017) reported that imidacloprid $17.8 \%$ SL @ 50 a. i. g/ha and 37.5 a.i. g/ha and recorded maximum reduction of thrips on chilli recording $96.13 \%$ and $94.96 \%$ respectively at one day after spray. Das et al., (2010) reported that a rapid degradation of persistency was observed in Imidacloprid which had a greater importance as fruits and vegetables are consumed after little cooking. Acharya et al., (2002) reported that the efficacy of new molecules like imidacloprid, abamectin were safer to lady bird beetles. These supported the present findings.

\section{Acknowledgements}

This study was carried out with the support of the Director of research and Department of Agricultural Entomology, BCKV; I thank the Department, and those who have contributed to it. 


\section{References}

Abbott, W. S. (1925). A method of computing the effectiveness of an insecticide. J.Econ. Entomol., 18: 265-267.

Acharya, S., Mishra, H.P. and Dash, D. (2002). Efficacy of insecticides against okra jassid (Amrasca biguttula biguttula Ishida). Annals of Plant Protection Science, 10(2): 230-232.

Bala, S.C., Karmakar, K. and Ghosh, S.K. (2015). Population dynamics of mite , Aceria tulipae Keif. On garlic (Allium sativum L.) and its management under Bengal basin. International Journal of Science, Environment and Technology. 4(5): 1365-1372.

Chakraborty, K. and Ghosh, S.K. (2010). Incidence of Coccinella septempunctata in brinjal with some pesticides. Current advances in Agricultural Sciences, 2(2): 129-130.

Das, K., Biswas, S., Chakraborty,G. and Ghosh, S.K. (2010). Efficacy of insecticides against Iassid (Amrasca biguttula biguttuka Ishida) on okra in terai agro-ecology of West Bengal. $J$. Appl. Zool. Res., 21(1): 33-35.

Ghosh, S.K.(1999). Studies on the pest constraints of brinjal/eggplant (Solanum melongena L.) and their management under terai region of West Bengal, India. $\mathrm{Ph} . \mathrm{D}$. Thesis awarded by BCKVAgriculture University, West Bengal, India. 1999; Pp. 43-44.

Ghosh, S.K (2013) Harmful effect of insecticides in the population dynamics of spiders on lady's fingers Abelmoschus esculentus (L.) Moench at field level. American-Eurasian Journal of Agricultural \& Environmental Sciences, 13 (9): 1181-1186.

Ghosh, S.K. (2013) Sustainable management of mango hopper (Amritodas atkinsony Leth. and Idioscopus niveosparsus Leth. Together) by using insect growth regulator (IGR), buprofezin 25 SC. Uttar Pradesh J. Zool., 33(2): 119-128.

Ghosh, S.K. (2020). Evaluation of safe insecticides against sucking pests, jassid (Amrasca bigutula bigutula Ishida) and aphid (Aphis gossypii Glov.) infesting chilli (Capsicum annum L.) crop. Journal of Entomology and Zoology studies, 8(5): 1428-1433.

Ghosh, S.K. and Senapati, S.K. (2003). Biology and seasonal abundance of jassid infesting brinjal/eggplant in terai region of West Bengal. Environment and Ecology, 21(3): 716-719.

Ghosh, S.K. and Chakraborty, K. (2012). Incidence and abundance of predatory beetle with special reference to Coccinella septempunctata in subHimalayan region of north-east India. International Journal of Plant, Animal and Environmental Sciences, 2(3): 157162.

Ghosh, S.K. and Chakraborty, K. (2015). Integrated field management of jassid (Amrasca biguttula biguttula Ishida.) infesting ladysfinger Abelmoschus esculentus (L.) Moench using biopesticides. International Journal of Science, Environment and Technology. 4 (2): 459-467.

Ghosh, S.K., Laskar, N. and senapati, S.K. (2005). Seasonal fluctuation of thrips on brinjal and field evaluation of pesticides. Pest Management and Economic Zoology. 13(2): 185-190.

Ghosh, S.K., Mahapatra, G.S.S. and Chakraborty, G.(2009). Field efficacy of plant extracts and microbial insecticides against aphid (Aphis gossypii) infesting okra (Abelmoschus esculentus). Redia, Itali XC11: 249-252. (with sub-title Journal of Entomology).

Ghosh, S.K., Mandal, T. and Chakraborty, K. (2013). Efficacy of chemical insecticides and neem oil against white fly (Bemisia tabaci Genn.) Infesting 
ladysfinger (Abelmoschus esculentus L.). International Journal of Bioresource and Stress Management 4 (2): special 348-351.

Ghosh, S.K., Mandol, T. and Chakraborty, K. (2016). Population fluctuation of aphid (Aphis craccivora Koch.) infesting Som plant leaves (Machilus bombycina King.) and its management. J. Ent.Res.. 40 (3): 235-241.

Ghosh, S.K., Laskar, N. and senapati, S.K. (2007). Seasonal incidence of predator Menochilus sexmaculatus Berliner on brinjal and harmful effect of insecticides on the predator. Indian J. Agric. Res 41(2): 102-106.

Ghosh, S.K., Mandal, T., Biswas, S. and Chakraborty, K. (2012). Field evaluation of cultivars and bio-efficacy of insecticides against pest complex of ladysfinger (Abelmoschus esculentus L.). Journal of applied Zoological research 23(2): 121-128.

Karmakar, K. and Bala, S.C. and Ghosh, S.K. (2017). Population dynamics of sheath mite (Stenotarsonemus spinki) Infesting rice cultivar IET-4786 and its management under Gangetic basin of West Bengal. Journal of Entomology and Zoology Studies, 5(4): 663-666.

Laskar, N. and Ghosh ,S.K, (2005). Efficacy of pesticides on the incidence of thrips, Scirtothrips dorsalis Hood and mite Polyphagotarsonamus latus Bank on chilli. J Plant Proto Environ. 2(2): 8083.

Mandol, T., Ghosh, S.K. and Chakraborty, K. (2016). Seasonal incidence of thrips (Thrips tabaci L.) infesting Som plant leaves (Machilus bombycina King.) and their management using bio-pesticides International Journal of Science, Environment and Technology. 5(4): 2245-2256.

Nayar, K.K., Ananthakrishnan, T.N. and David, B.V.(1992) General and Applied
Entomology, 430-431.

Priyadarshini, S., Pal, S. and Ghosh, S.K. (2017). Field screening of chilli cultivars against thrips (Scirtothrips dorsalis Hood.) and its management under West Bengal condition. Journal of Entomology and Zoology studies, 5(6): 2106--2110.

Priyadarshini, S., Ghosh, S.K. and Nayak, A.K. (2019). Field screening of different chilli cultivars against important sucking pests of chilli in West Bengal. Bulletin of Environment, Pharmacology and Life Sciences, 8(7): 134-140.

Saini, A., Ahir, K.C., Rana, B.S. and Kumar, R. (2017). Population dynamics of sucking pests infesting chilli (Capsicum annum L.). Journal of Entomology and Zoology Studies. 5: 250-252.

Subba, B., Pal, S., Mandal, T., Ghosh, S.K. (2017) Population dynamics of white fly (Bemisia tabaci Genn.) Infesting tomato (Lycopersicon esculentum L.) and their sustainable management using biopesticides. Journal of Entomology and Zoology Studies, 5(3): 879-883.

Subba, B. and Ghosh, S.K. (2016). population dynamics of lady bird beetle and spiders in relation to weather factors in tomato (lycopersicon esculentum 1.). Life Sciences International Research Journal, 3(1): 35-37.

Subba, B. and Ghosh, S.K. (2016). Population dynamics of Thrips (Thrips tabaci L.) Infesting tomato (Lycopersicon esculentum L.) and their sustainable management. International J. Agric. Sc. And Res, 6 (3): 473-480.

Subba, B. Ghosh, S.K., Banerjee, D. and Jasudasu, G.S.(2015). Seasonal incidence of Jassid Infesting tomato (Lycopersicon esculentum L.) and their sustainable management .Annals of Plant and Soil Research 17: 19-22. Special Issue).

Thakoor, P., Ghosh, S.K. Nihal, R. and 
Ramya Sri, N. (2019). Effect of abiotic factors on seasonal incidence and bioefficacy of some newer insecticides against aphid (Aphis gossypii) in tomato (Abelmoschus esculentus). Journal of Entomology and Zoology studies, 7(3): 513-516.

Thakoor, P., Ghosh, S.K. and Bala, S.C.
(2020) Effect of abiotic factors on seasonal incidence and bio-efficacy of some newer insecticides against white fly on tomato crop in West Bengal. Journal of Entomology and Zoology studies. 8(3): 267-271.

\section{How to cite this article:}

Sunil Kumar Ghosh. 2020. Management of Sucking Pest, Jassid (Amrasca devastans) and Thrips (Thrips palmi) on Lady's Finger (Abelmoschus esculentus L.) by using Safe Insecticides. Int.J.Curr.Microbiol.App.Sci. 9(11): 2340-2352.

doi: https://doi.org/10.20546/ijcmas.2020.911.281 